\title{
Treatment of alcohol use disorders in the elderly: an overview of RCTs
}

In this issue of International Psychogeriatrics, our research group published a paper on alcohol use disorders (AUD) in elderly adults (Kist et al., 2014). According to different authors, age of onset is an important characteristic to distinguish subgroups of patients with AUD, similar to, for example, late-onset depression (LOD) and lateonset schizophrenia (LOS). The cut off age to discriminate between early and late-onset AUD varies but is often 25 years, which is substantially younger than the cutoffs to discriminate LOD or LOS. However, in AUD, many patients started having alcohol problems much later in life, for example, after 45,50 , or even after 60 years. These very late onset patients are almost completely ignored in research. In a group of older inpatients with AUD, we found that those who started drinking heavily after 25 and after 45 years of age have similar cognitive impairments compared with their peers who started drinking before 25 years of age. Our results are counter-intuitive at first instance, but some other authors have found the same results. However, several potential limitations may also account for this result, and replication is needed before conclusions that are more definite can be drawn. In the preparation of this editorial, we found a lack of research in patients who started drinking excessive amounts of alcohol very late in life. This is in contrast with, for example, a fair amount of research in LOD and at least some research in LOS.

The lack of research cannot be explained by a low frequency or importance of AUD in the elderly using mental health care. The 12-month prevalence of AUD was $1.5 \%$ in a large epidemiological study of adults aged 65 years and older $(N>$ 8,000 ), with a lifetime prevalence of $16.1 \%$ ( $\mathrm{Lin}$ et al., 2011). Another recent large epidemiological study $(N>16,000)$ has found an estimated 12-month prevalence of alcohol dependence of $0.6 \%$, with a $0.9 \%$ prevalence of alcohol abuse in community-living elderly adults aged 65 years and over (Blazer and Wu, 2011). However, not only AUD may be relevant, sub-threshold disorders and at-risk or binge drinking should also be taken into account. The 12-month prevalence of subthreshold dependence was $5.2 \%$ in the study done by Blazer and Wu (2011). In the same study population, at-risk drinking was reported by $13 \%$ of men and $8 \%$ of women, and more than $14 \%$ of men and 3\% of women reported binge drinking (Blazer and $\mathrm{Wu}, 2009)$. In general hospitals up to $30 \%$ of older patients and in psychiatric hospitals up to $50 \%$ of older patients were diagnosed with AUD (Caputo et al., 2012). In nursing homes, the prevalence of AUD or drinking problem varies between $8 \%$ and 53\% (Johnson et al., 2000). Chronic alcohol use is associated with multiple physical diseases and psychiatric disorders, including depression, anxiety, and dementia (Caputo et al., 2012). Alcohol causes approximately $3.8 \%$ of all deaths and $4.6 \%$ of the global burden of disease worldwide (Rehm et al., 2009). These papers on prevalence were published in major (geriatric) psychiatric journals, and many healthcare workers will be familiar with the results.

If we were not be aware of how to screen or diagnose elderly patient with AUD, then this could be a potential explanation for lack of research in this field. Excellent systematic reviews have been published on the screening instruments for AUD in elderly people (Berks and McCormick, 2008; O'Connell et al., 2004), both suggesting the Alcohol Use Disorders Identification Test (AUDIT; Babor et al., 2001) as a useful screen, especially to detect harmful and hazardous drinking in the elderly. With regard to CAGE (Cut down, Annoyed, Guilty and Eye-opener), there was no agreement between the above-cited two reviews.

Another potential explanation would be that healthcare workers are not aware of treatment studies, and consider elderly patients with AUD as hopeless. Personally, I was also not aware of any systematic review of treatment studies on AUD in the elderly, and was familiar with only a few randomized controlled trials (RCTs) published in (geriatric) psychiatric journals. A literature search revealed two recent reviews of treatment studies, but neither of these was published in a (geriatric) psychiatric journal, and the same is valid for many for the RCTs that were included in aforementioned reviews. Perhaps old age psychiatrists and psychologists treating elderly adults with AUD are not aware of these reviews and RCTs.

The first of the aforementioned reviews is, according to its title, a systematic and narrative review of treatment for older people with substance problems (Moy et al., 2011). This review found only six studies with an extensive research strategy, 
but ten additional studies included in the review were obtained from expert advisors and background research, suggesting that finding the evidence base of treatment of AUD in the elderly is not easy. Eleven of these studies were related to alcohol dependence, of which only three were RCTs. This review concluded that older people do respond to treatment and do not achieve worse outcomes than younger adults, sometimes even better results.

Interestingly, the second review published only two years later found 12 RCTs of treatment for AUD (Kuerbis et al., 2013). These authors concluded that treatment generally works for the elderly, yielding rates of abstinence comparable with general populations and younger patient groups. Age-specific treatments appeared to enhance treatment effects for older adults. One study (Oslin, 2005), identified by Moy et al. (2011), was not included in the review by Kuerbis et al. (2013). With an additional literature search for the preparation of this editorial, two RCTs were found that have been published very recently (Ettner et al., 2014; Vrdoljak et al., 2014). Thus, at least $15 \mathrm{RCTs}$ are published on the treatment of AUD (see reference lists of all RCTs).

Three of these RCTs studied naltrexone versus placebo (Oslin et al., 1997; 2002; Oslin, 2005). No statistically significant differences in abstinence rates were found between naltrexone and placebo, but sample sizes were small in these three studies. However, abstinence was achieved by the majority of patients, and rates were higher in the elderly compared with younger adults (Oslin et al., 2002).

Brief interventions in a primary care population were studied in three RCTs (Fleming et al., 1999; Gordon et al., 2003; Fink et al., 2005). These interventions were more effective than care as usual (CAU) in two of these RCTs, the sample size of the negative RCT was probably too small to detect statistically significant differences.

Interdisciplinary, broad, or multifaceted mental healthcare management programs, either with primary care patients or male veterans, were studied in more RCTs (Bartels et al., 2004; Oslin et al., 2004; Moore et al., 2010; Ettner et al, 2014; Vrdoljak et al., 2014). Disappointingly, only two RCTs (Moore et al., 2010; Ettner et al., 2014) found a statistically significant advantage of intervention over CAU. However, in all but one trial, the outcome variable number of drinks, number of patients drinking at-risk, and score on AUDIT) decreased significantly in both intervention and CAU groups. The negative trial was not primarily aimed to reduce alcohol consumption, but to improve physical activity, smoking, alcohol consumption, and diet (Vrdoljak et al., 2014). One telephone-based disease management program aimed at primary care patients was not statistically significant compared with CAU either, probably due to small sample sizes (Oslin et al., 2003).

Two RCTs comparing different approaches are especially interesting. In an older RCT, an alcoholic rehabilitation program operated by a tolerant and supportive staff specialized in treating elderly alcoholics was more effective in achieving abstinence than a more traditional program emphasizing confrontation with patients' past failures and present conflicts (Kashner et al., 1992). Abstinence rates increased as patient's age increased: at 12 months, the odds of reporting abstinence increased 2.5 times for every ten years of patient's age. Another important RCT found that an individual, medically oriented and integrated care condition using a harm-reduction approach was more effective in reducing the number of drinks and the number of binge-drinking episodes than a community-based group program using the 12-step model of total abstinence (Lee et al., 2009).

At first glance, there appears to be a fair number of RCTs on treatment of alcohol-related problems in the elderly. Only a minority of these RCTs have used Diagnostic and Statistical Manual of Mental Disorders (DSM) or International Classification of Diseases (ICD) criteria to define AUD (Rice et al., 1993; Oslin et al., 1997; 2002; Oslin, 2005). However, it is debatable as to what extent these criteria can be applied in elderly patients. A wide variety of measures were used to include study patients, but objective outcome measures (e.g. blood tests) were rarely used. In addition, studies differ greatly from each other in the cut off age to define older people (45-65 years old), the number of older patients included (34-2,637), settings (primary care, outpatients, or inpatients), and study duration (12 weeks-24 months). The heterogeneity is also emphasized in the review of Moy et al. (2011). It is particularly disappointing that RCTs of pharmacological treatments other than naltrexone are lacking. Acamprosate, disulfiram, baclofen, topiramate, and nalmefene have not been studied in RCTs in the elderly, nor any subgroup or post hoc analyses in elderly patients have been published. Controlled studies evaluating the efficacy of medication for the treatment of alcohol withdrawal symptoms in older persons are also lacking.

Only six of the 15 RCTs found statistically significant differences between the intervention under study and CAU, although some studies were clearly underpowered. However, taking a closer look at the RCTs reveals that usually a high number of patients achieved decrease in alcohol consumption or remission, sometimes even with 
minimal interventions. Unfortunately, based on these RCTs, we cannot conclude which treatment works as the best for older adults and why. There are no RCTs with a stepped care approach, offering more intensive treatment after failure of the first line therapy (e.g. a minimal intervention). E-health studies are completely lacking as well. No study reported age to be a significant negative predictor of outcome. Four RCTs presented age as outcome predictor: two studies found no difference between older and younger adults (Rice et al., 1993; Gordon et al., 2003) and two studies found age to be a predictor of a better outcome (Kashner et al., 1992; Oslin et al., 2002). Only one RCT analyzed the subgroup of elderly that started drinking too much alcohol very late in life (after 40 years of age) and found lower alcohol consumption at followup compared with persons with an earlier onset of alcohol-related problems (Fleming et al., 1999). In addition to these RCTs, many cohort studies have compared older age groups with younger age groups and found either no difference in prognosis, or sometimes even better results in the elderly (Moy et al., 2011; Kuerbis et al., 2013). This may in part be the result of elderly persons being more motivated and/or staying in treatment for a longer period, or having less severe AUD. This may also explain the better prognosis of women with AUD found in some studies in comparison with men.

In conclusion, there is no evidence base for treating elderly patients with an AUD, at-risk drinking, or problematic drinking in a different way than younger adults with the same disorders or the same level of alcohol use. Elderly persons respond to treatment as well and perhaps even better compared with younger adults. Age-specific treatments may work better in the elderly than treatment in mixed age groups. Age of onset is understudied in RCTs, and whether patients with a (very) late age of onset have a different prognosis and need specialized treatment, tailored to different etiologies of their AUD, is not known.

\section{Conflict of interests}

None

RoB M KOK

Department of Old Age, Parnassia Psychiatric Institute, Mangostraat 1, 2552 KS The Hague, The Netherlands Email: r.kok@parnassia.nl

\section{References}

Babor, T. et al. (2001). AUDIT. The Alcohol Use Disorders Identification Test. Guidelines for Use in Primary Care, 2nd edn. Geneva, Switzerland: World Health Organization.

Bartels, S. J. et al. (2004). Improving access to geriatric mental health services: a randomized trial comparing treatment engagement with integrated versus enhanced referral care for depression, anxiety, and at-risk alcohol use. American fournal of Psychiatry, 161, 1455-1162.

Berks, J. and McCormick, R. (2008). Screening for alcohol misuse in elderly primary care patients: a systematic literature review. International Psychogeriatrics, 20, 1090-1103.

Blazer, D. G. and Wu, L. T. (2009). The epidemiology of at-risk and binge drinking among middle-aged and elderly community adults: National Survey on Drug Use and Health. American fournal of Psychiatry, 166, 1162-1169.

Blazer, D. G. and Wu, L. T. (2011). The epidemiology of alcohol use disorders and sub-threshold dependence in a middle-aged and elderly community sample. American Fournal of Geriatric Psychiatry, 19, 685-694.

Caputo, F., Vignoli, T., Leggio, L., Addolorato, G., Zoli, G. and Bernardi, M. (2012). Alcohol use disorders in the elderly: a brief overview from epidemiology to treatment options. Experimental Gerontology, 47, 411-416.

Ettner, S. L. et al. (2014). The effect of an educational intervention on alcohol consumption, at-risk drinking, and health care utilization in older adults: the Project SHARE study. Fournal of Studies on Alcohol and Drugs, 75, 447-457.

Fink, A., Elliot, M. N., Tsai, M. and Beck, J. C. (2005). An evaluation of an intervention to assist primary care physicians in screening and educating older patients who use alcohol. Fournal of the American Geriatrics Society, 53, 1937-1943.

Fleming, M. F., Manwell, L. B., Barry, K. L., Adams, W. and Stauffacher, E. A. (1999). Brief physician advice for alcohol problems in older adults: a randomized community-based trial. Fournal of Family Practice, 48, 378-384.

Gordon, A. J., Conigliaro, J., Maisto, S. A., McNeil, M., Kraemer, K. L. and K. L., Kelley, M. E. (2003). Comparison of consumption effects of brief interventions for hazardous drinking elderly. Substance Use and Misuse, 38, 1017-1035.

Johnson, I. (2000). Alcohol problems in old age: a review of recent epidemiological research. International fournal of Geriatric Psychiatry, 15, 575-581.

Kashner, M., Rodell, D. E., Ogden, S. R., Guggenheim, F. G. and Karson, C. N. (1992). Outcomes and costs of two VA inpatient treatment programs for older adult alcoholic patients. Hospital and Community Psychiatry, 43, 985-989.

Kist, N., Sandjojo, J., Kok, R. M. and Van den Berg, J. F. (2014). Cognitive functioning in older adults with early, late, and very-late onset alcohol dependence. International Psychogeriatrics. http://dx.doi.org/10.1017/ S1041610214000878.

Kuerbis, A. and Sacco, P. (2013). A review of existing treatments for substance abuse among the elderly and recommendations for future directions. Substance Abuse, 13, 13-37.

Lee, H. S., Mericle, A. A., Ayalon, L. and Areán, P. A. (2009). Harm reduction among at-risk elderly drinkers: a site-specific analysis from the multi-site primary care 
research in substance abuse and mental health for elderly (PRISM-E) study. International fournal of Geriatric Psychiatry, 24, 54-60.

Lin, J. C. et al. (2011). Alcohol, tobacco, and non-medical drug use disorders in US Adults aged 65 years and older: data from the 2001-2002 National Epidemiologic Survey of Alcohol and Related Conditions. American fournal of Geriatric Psychiatry, 19, 292-299.

Moore, A. A. et al. (2010). Primary care-based intervention to reduce at-risk drinking in older adults: a randomized controlled trial. Addiction, 106, 111-120.

Moy, I., Crome, P., Crome, I. and Fisher, M. (2011). Systematic and narrative review of treatment for older people with substance problems. European Geriatric Medicine, 2, 212-236.

O'Connell, H. et al. (2004). A systematic review of the utility of self-report alcohol screening instruments in the elderly. International Fournal of Geriatric Psychiatry, 19, 1074-1086.

Oslin, D. W. (2005). Treatment of late-life depression complicated by alcohol dependence. American fournal of Geriatric Psychiatry, 13, 491-500.

Oslin, D. W., Liberto, J. G., O’Brien, J., Krois, S. and Norbeck, J. (1997). Naltrexone as an adjunctive treatment for older patients with alcohol dependence. American Fournal of Geriatric Psychiatry, 5, 324-332.
Oslin, D. W., Pettinati, H. and Volpicelli, J. R. (2002). Alcoholism treatment adherence: older age predicts better adherence and drinking outcomes. American fournal of Geriatric Psychiatry, 10, 740-747.

Oslin, D. W. et al. (2003). Disease management for depression and at-risk drinking via telephone in an older population of veterans. Psychosomatic Medicine, 65, 931-937.

Oslin, D. W. et al. (2004). Treatment effects from UPBEAT: a randomized trial of care management for behavioral health problems in hospitalized elderly patients. Fournal of Geriatric Psychiatry and Neurology, 17, 99-106.

Rehm, J., Mathers, C., Popova, S., Thavorncharoensap, M., Teerawattananon, Y. and Patra, J. (2009). Global burden of disease and injury and economic cost attributable to alcohol use and alcohol-use disorders. Lancet, 373, 2223-2233.

Rice, C., Longabaugh, R., Beattie, M. and Noel, N. (1993). Age group differences in response to treatment for problematic alcohol use. Addiction, 88, 1369-1375.

Vrdoljak, D. et al. (2014). Lifestyle intervention in general practice for physical activity, smoking, alcohol consumption and diet in elderly: a randomized controlled trial. Archives of Gerontology and Geriatrics, 58, 160-169. 